\title{
Evaluaciones de impacto social. La antropología en la elaboración de proyectos de la Comisión Federal de Electricidad en México
}

\author{
Social Impact Assessments. The a nthropology in the \\ preparation of projects of the Federal Electricity \\ Commission in Mexico
} iD Janet Silvia Salazar Patiño; ${ }^{1}$ José Luis Arriaga Ornelas²

\begin{abstract}
Resumen
En México el servicio de energía eléctrica es prestado de manera casi-monopólica por la Comisión Federal de Electricidad -CFE-, que es en una "empresa productiva del Estado" y presenta tendencias de crecimiento sostenidas, lo cual le lleva a emprender proyectos de infraestructura permanentemente. El nuevo marco normativo para realizar esos proyectos le obliga a realizar Evaluaciones de Impacto Social previas. Este trabajo describe la inclusión de algunos antropólogos en la realización de las primeras evaluaciones, también se comparte la vivencia directa en algunos de ellos y se reflexiona sobre el tipo de rol que se le pide jugar. Se expone cómo se están llevando a cabo las evaluaciones y se ofrecen datos recabados durante proyectos de subestaciones y nuevas líneas de transmisión en la región de Silao, Guanajuato, en donde se documentó que la gente se resiste a los proyectos, ya sea por desconfianza o como estrategia para obtener la mejor ganancia. Finalmente se reflexiona acerca de la posición del antropólogo, las limitaciones que ahora se le imponen en este tipo de estudios y las potencialidades que tendría como mediador y evaluador de los proyectos, sin soslayar la disyuntiva deontológica al colaborar en proyectos e influir en las decisiones que orienten las acciones planificadas de la CFE.
\end{abstract}

Palabras clave: Evaluación de impacto social; antropología; energía eléctrica; Comisión Federal de Electricidad.

Tipología: Artículo de Investigación.

Recibido: 30/06/2018

Evaluado: $27 / 01 / 2019$

Aceptado: 12/03/2019

Disponible en línea: 18/03/2019

Como citar este artículo: Salazar-Patiño, Janeth S. \& Arriaga-Ornelas, José L. (2019). Evaluaciones de impacto social. Los antropólogos en la elaboración de proyectos de la Comisión Federal de Electricidad en México. Jangwa Pana, 18 (1) 72 - 87. Doi: http://dx.doi.org/10.21676/16574923.2779

\footnotetext{
${ }^{1}$ Licenciada en antropología social. Universidad Autónoma del Estado de México. Orcid ID: 0000-0002-4589-2394.

Correo electrónico: janiiz_salazar@hotmail.com

${ }^{2}$ Doctor en ciencias sociales. Universidad Autónoma del Estado de México. Orcid ID: 0000-0002-0498-8461. Correo electrónico: jlarriagao@gmail.com
} 


\begin{abstract}
In Mexico, the electric power service is provided by the Federal Electricity Commission (CFE). For three years, it has been a "productive State enterprise", with sustained growth, which leads it to undertake infrastructure projects all the time. There are new laws that require you to carry out Social Impact Assessments in each project. This paper describes the way anthropology finds space to collaborate in these studies. The guidelines followed by the CFE for the preparation of the studies are analyzed and the way the evaluations are being carried out is described. Empirical data are provided during substation projects and new transmission lines in the Silao region, Guanajuato. It documents the way in which people resist projects, whether due to distrust or as a strategy to obtain the best compensation. Finally, we reflect on the position of the anthropologist, as mediator and endorsement of the projects.
\end{abstract}

Key words: Social impact assessment; anthropology; electric power; Federal Electricity Commission

\section{Introducción}

Toda intervención, planificada o no, que se realiza en una sociedad genera cambios, tanto en la sociedad como en el mismo proceso de intervención. La promoción del Desarrollo es una intervención, y por tanto genera cambios y está sometida a cambios. Sin embargo, por décadas, los programas y proyectos promotores del desarrollo no se interesaron por generar conocimiento sobre su previsible impacto en el ámbito social. No fue sino hasta el último tercio del siglo pasado que comenzó a atenderse un interés por proyectar, analizar, monitorear e incluso gestionar las consecuencias sociales del Desarrollo (Esteves, Franks y Vanclay, 2012; Becker, 1997; Vanclay, 1999). Aunque ello vino un poco después que la normatividad de distintas naciones estableció la obligatoriedad de proyectar el impacto ambiental de los proyectos. Enseguida se hizo evidente que, así como se suele incidir en el paisaje o la biota de una región, cualquier proyecto encerraba potenciales impactos sociales y se asumió que era posible preverlos e incluso emprender acciones de mitigación y control, sobre todo de los impactos negativos que sobre los grupos humanos puedan ocasionar las actividades de desarrollo (McPherson, 2004).

En México las Evaluaciones de Impacto Social -EIS- son más bien algo nuevo. La ley ha previsto que distintos proyectos, en materia de desarrollo y extracción de recursos, efectúe este tipo de trabajos, pero su insipiencia hace evidente que apenas está en construcción un corpus de conocimientos teóricos y metodológicos en esta materia. Hay, desde luego, un volumen mayor de experiencias y conocimiento acumulado en materia de evaluación de impacto de programas públicos (García y Cardozo, 2017; Pichardo, 2013; CONEVAL, 2014). Pero existe una diferencia entre ambos tipos de estudio: el primero busca efectuar una evaluación prospectiva de las consecuencias sociales que pueden ser derivadas de acciones políticas, programas y del desarrollo de proyectos, en un particular contexto (Burdge y Vanclay, 1995). En tanto que el segundo, apunta hacia el diagnóstico de las acciones y resultados en el marco de una intervención planificada (Pichardo, 2013).

Igualmente hay una diferencia entre este tipo de estudios y los de Antropología Aplicada, lo cual es preciso aclarar de inicio, porque el presente trabajo no es precisamente una propuesta para la aplicación de conocimientos antropológicos en el sentido capacidad técnica para conducir proyectos (Foster, 1964), sino que es un examen sobre el tipo de uso del trabajo antropológico que hace un promotor del Desarrollo (en este caso la CFE). Esto último más bien se inscribiría en el ámbito de la "antropología práctica" a la que se refirió Malinowski, con su muy referido trabajo "Practical Anthropology" publicado en 1929, en donde hacía una abierta invitación a las 
agencias gubernamentales para apelar a los antropólogos cuando necesitaran ayuda.

Esta es la razón por la cual el presente artículo ubica su labor descriptiva en las nuevas obligaciones legales que existen en México para que, antes de emprender un proyecto (sobre todo energético, porque así lo mandata el marco jurídico), se identifiquen y evalúen los efectos que tendría el mismo en el ámbito social. Los datos que se ofrecen provienen de la participación de la autora en las primeras EIS que se ha visto obligada a hacer la CFE por ser la empresa productiva del Estado mexicano que posee el más amplio segmento del mercado de energía eléctrica y que sigue en crecimiento, promoviendo nuevos proyectos, $\mathrm{y}$ que ahora por mandato legal deben estar precedidos de EIS. Se buscará resaltar que las obligaciones que ahora acata la CFE no vinieron precedidas por una reflexión epistemológica sobre las consecuencias del Desarrollo, pues que se plantearan el problema de lo social o lo cultural como materia que debía ser tomada en cuenta antes de iniciar nuevos proyectos, se genera por una disposición normativa.

El trabajo tiene un carácter descriptivo, porque se desprende de la participación de la autora en las primeras EIS que realizó la CFE, específicamente en el estado de Guanajuato, México. Metodológicamente se procedió a reunir los elementos derivados de esa experiencia (donde el trabajo de campo tuvo un papel central), para así reflexionar acerca de ellos a la luz del conocimiento sobre las EIS y a ponderar el rol que se está asignando específicamente a los antropólogos en este tipo de estudios en el caso de la CFE. Derivado de ello, el texto se compone de tres partes: la primera, ubica a las EIS en el tiempo y su evolución hasta constituirse en una subdisciplina científica a nivel internacional. La segunda, ofrece un panorama del trabajo realizado por la CFE y su nuevo marco regulatorio, en el que se incluye la figura de las EIS; y por último, se comparte una experiencia directa tenida desde la CFE en un proyecto realizado en el estado de Guanajuato, en la zona centro de México. En la parte final se incluyeron algunas reflexiones sobre las condicionantes a que se enfrenta el antropólogo social para involucrarse de manera protagónica en la gestión de los problemas sociales que vienen aparejados con las intervenciones planificadas de la CFE.

\section{Las EIS, origen y transformación}

Las EIS son entendidas, de manera general, como un ejercicio predictivo, realizado en el marco de un proyecto que busca emprender acciones de intervención sobre una sociedad y/o su entorno. Se les ubica muy cerca de las evaluaciones o declaraciones de impacto ambiental, que comenzaron a realizarse en la segunda mitad del siglo pasado. Cuando empezó a esbozarse su campo de acción y procedimiento se subrayaban su utilidad técnica y su carácter científico. Respecto de lo primero, se afirmaban cosas como la siguiente:

es un proceso de investigación, en el que se precisan las consecuencias producidas por la ejecución de determinados programas y proyectos en determinadas condiciones. Al ser una investigación, se constituye en una operación de análisis, que implica recolectar información cuantitativa y cualitativa en forma sistemática (Kisnerman, 1984: 52).

Y respecto de lo segundo, se decía: "La evaluación, como actividad científica, debe ajustarse a los requisitos, las características y las metodologías definidas por la epistemología." (Gómez, 2004: 88). Debe subrayarse que desde su origen se pensó a las EIS como un trabajo técnico de alta especialidad y basado en metodologías científicas; no se trataba sólo de estimaciones políticas, ponderaciones ideológicas o éticas.

Conviene destacar también que la presencia de investigadores, proyectando potenciales afectaciones al paisaje, la biota y la vida social en el marco de un proyecto o programa, significó declarar que el Desarrollo implica problemas que era preciso prever y la ley, en muchas naciones, empezó a considerar la ponderación de tales afectaciones como una condicionante para la aprobación de un 
proyecto. Varios trabajos (Esteves, Franks y Vanclay, 2012; Burdge y Vanclay, 1995; Becker, 1997; Vanclay, 1999) coinciden al decir que las EIS aparecieron de manera formal, como requisito legal y como parte de la planificación de los proyectos, a partir de la promulgación en los Estados Unidos de América de la Ley de Política Ambiental Nacional -NEPA, por sus siglas en inglés- en el año de 1969.

Este primer momento de las EIS tiene una característica muy particular; se consideran actividades que se realizan por el interés de la agencia promotora del proyecto y/o de la instancia gubernamental que debe autorizarlo. Quienes las realizan son especialistas con habilidades técnicas para diagnosticar la condición inicial del contexto donde se proyecta y los potenciales escenarios a partir de la intervención. Vistas de ese modo, se convierten en un procedimiento unilateral encaminado a:

\begin{abstract}
identificar, obtener y proporcionar información útil y descriptiva acerca del valor y el mérito de las metas, la planificación, la realización y el impacto de un objeto determinado, con el fin de servir de guía para la toma de decisiones, solucionar los problemas de responsabilidad y promover la comprensión de los fenómenos implicados (Stufflebeam y Shinkfield, 1987:183).
\end{abstract}

El destinatario de la información generada va a ser, primariamente, la agencia $y$, en un segundo momento, la autoridad. Se entiende que dicha información es necesaria para tomar decisiones, hacer modificaciones o definir alternativas en un proyecto. En suma, sólo había una parte activa que emprendía el "proceso de reconocer las áreas de decisión importantes, seleccionar la información apropiada y reconocer y analizar la información para transmitir datos que ayudan a tomar decisiones para tomar alternativas" (Alkin, 1969, citado por Tenbrick, 1998: 2). Y quien hacía las EIS de cualquier proyecto debía tener en mente que se trataba de obtener información y usarla para expresar juicios, que a su vez se utilizarán en la toma de decisiones, siendo todo encaminado a prevenir, predecir, incrementar, identificar y comunicar los posibles efectos que se puedan presentar durante su ejecución y al término del mismo (Tenbrick, 1988). Será con el paso del tiempo que estas acciones unilaterales comiencen a ser pensadas más en términos dialogales, y ese será un segundo momento de las EIS.

Este segundo momento puede explicarse como un resultado casi natural de la delimitación de un campo de investigación, la inclusión en él de profesionales de distintas disciplinas, el trabajo en campo y la conformación de un discurso con la consecuente afirmación de un paradigma anclado en la gestión de los problemas sociales del desarrollo. La articulación de estos factores posibilitó que las EIS devinieran en la emergencia de la Social Impact Assessment -SIA-, a la que algunos denominan:

una ciencia social interdisciplinaria y/o transdisciplinaria que incorpora muchos campos incluyendo sociología, antropología, demografía, estudios de desarrollo, estudios de género, geografía social y cultural, economía, ciencias políticas y derechos humanos, psicología ambiental y comunitaria, métodos de investigación social y ambiental ley, entre otros (Esteves, Franks y Vanclay, 2012: 35).

La SIA se presenta ahora como un área del conocimiento que contribuye al proceso de Desarrollo, pero que no se detiene en el mero estudio técnico predictivo que resulta de utilidad para la agencia promotora del proyecto, o que es un requisito legal para la ejecución del mismo. La SIA hace una apuesta por trabajar de manera simultánea con tres tipos de actores: las comunidades, buscando lograr mejores resultados para ellas; las agencias de desarrollo y compañías, para contribuir al diseño de mejores proyectos; y con las agencias reguladoras o autoridades, para proporcionar información necesaria para 
la aprobación, regulación continua y evaluación de los proyectos (Vanclay, 2003).

Desde luego que este devenir de las EIS hacia un enfoque más dialogal no ha sido generalizado. En muchos países la inclusión de una obligatoriedad legal de efectuar las evaluaciones ha sido más bien correspondiente con el primer momento, con EIS destinadas a proveer de información a la agencia promotora y a la autoridad. En el caso que interesa a este trabajo y que se expondrá en las siguientes páginas, una reforma legal realizada en México para permitir la inclusión de la iniciativa privada en el campo energético, incluyó como requisito legal para cualquier nuevo proyecto estudios de impacto ambiental y social. La legislación secundaria, en la cual se dice cómo se deben hacer esas evaluaciones piensa las EIS como:

Un documento que contiene la identificación de las comunidades y los pueblos ubicados en el área de influencia de un proyecto del sector energético, así como la identificación, caracterización, predicción y valoración de las consecuencias a la población que podrían derivarse del mismo y las medidas de mitigación y los planes de gestión social correspondiente (COFEMER, 2015: 4).

Buena parte de la discusión que puede derivarse de primar el enfoque unilateral o el dialogal reside en preguntas como las siguientes: ¿quién necesita saber el impacto social que podría causar un proyecto; la agencia promotora, la autoridad(es) involucrada o la comunidad(es) impactada? ¿El impacto social se estima desde la perspectiva del evaluador, de la agencia promotora $\mathrm{o}$ de los potencialmente impactados? ¿La información generada debe ser transmitida a todos por igual o se necesita procesar de modo diferenciado para unos y otros? ¿Es labor de quien elabora las EIS erigirse como traductor de unos y otros, como mediador, como conciliador?

\section{Viejas y nuevas dinámicas de la industria eléctrica en México}

Actualmente en México el servicio de energía eléctrica lo ofrece de manera cuasi-monopólica la Comisión Federal de Electricidad -CFE-, entidad que se encuentra subordinada a la Secretaría de Energía -SENER-, que es una dependencia del Poder Ejecutivo Federal cuya responsabilidad es establecer la política energética del país. La condición histórica que tenía la CFE, de ser la única instancia facultada para generar, transmitir, distribuir y comercializar la energía eléctrica en el país, dejó de existir tras una serie de reformas a la Constitución, a las que se les conoció informativamente como "Reforma Energética". A partir del año 2015 este nuevo marco normativo obligó a la CFE a transformarse en una "empresa productiva del Estado" y a disponerse a competir con empresas nacionales y extranjeras en el mercado energético (CFE, 2015).

No obstante, todavía cuenta con la mayoría del mercado, 39 millones de clientes, lo que representa aproximadamente 121 millones de habitantes del país entero. Justo antes de convertirse en empresa productiva del Estado reportó haber suministrado el servicio a más de 150 mil localidades, de las cuales 146,164 son consideradas rurales y 4,049 urbanas (CFE, 2015).

En México el porcentaje de clientes nuevos para la CFE crece anualmente entre 800 mil a 1 millón, de los cuales el $88 \%$ son usuarios de tipo doméstico. El servicio de energía eléctrica creció en los últimos años hasta llegar casi a la cobertura total del territorio nacional, permitiendo que la CFE haya presentado un aumento de 2.4 millones referente al número de viviendas con el servicio en comparación con años anteriores (CFE, 2018). Actualmente este servicio está llegando al $98.7 \%$ de los hogares mexicanos, de acuerdo a la información proporcionada por la encuesta intercensal de INEGI (2015).

En el nuevo marco de competencia por el mercado, la CFE busca presentarse como "una 
empresa rentable de clase mundial que participa competitivamente en la satisfacción de la demanda del servicio público de energía eléctrica nacional e internacional" (García, 2007:54). Esto no deja lugar a dudas respecto al modelo de desarrollo energético que anima a una necesidad de permanentes obras en materia de electrificación (Barabás y Bartolomé, 1992). Por esta razón, es muy importante poner atención a las condiciones en las que proyectan y ejecutan dichas obras.

Obligada por un nuevo marco regulatorio para su operación, la CFE asegura prestar el servicio público de energía eléctrica tomando en cuenta una serie de criterios de suficiencia, competitividad y sustentabilidad. En términos operativos, la CFE cuenta para la generación de energía con centrales termoeléctricas, hidroeléctricas, carboeléctricas, geotermoeléctricas, eoloeléctricas y nucleoeléctrica, y para conducir la electricidad desde las centrales de generación hasta los hogares de cada uno de sus clientes, cuenta con 874 mil kilómetros de líneas de transmisión por todo el país (Serrano, 2011).

Para este artículo interesa destacar que los trabajos de expansión de la CFE en territorio nacional son permanentes. Organizacionalmente dicha empresa cuenta con una Coordinación de Proyectos de Transmisión y Transformación -CPTT-, que forma parte de la Subdirección de Proyectos y Construcción de la Dirección de Proyectos de Inversión Financiada, instancia encargada de desarrollar y construir los Proyectos de Subestaciones Eléctricas y Líneas de Transmisión de alta tensión en todo el territorio nacional. El interés de subrayarlo es porque desde estas oficinas se planean y ejecutan proyectos en los que la empresa entra en

\footnotetext{
${ }^{3}$ Con fecha 1 de julio de 1990, C.F.E. y su sindicato de trabajadores (el S.U.T.E.R.M.) celebran el convenio C.F.E./S.U.T.E.R.M. 074/90, en el cual quedan constituidas las Residencias Generales de Construcción, conviniendo en que las áreas desconcentradas dependan jerárquicamente y funcionalmente de la Gerencia de Construcción, en razón de las obras a su cargo y de acuerdo a su ubicación geográfica, quedando establecidas las Residencias Regionales de área de Proyectos de Transmisión y Transformación" (Xolo 2011:20)
}

contacto con las comunidades, ante la necesidad de emprender nuevos planes y que en su gran mayoría implican afectaciones, sobre todo territoriales, en regiones rurales, semiurbanas $\mathrm{y}$, en ocasiones, absolutamente marginales.

El personal de la empresa que lleva a cabo estas labores está distribuido dentro del territorio nacional con base en Residencias Generales de Construcción de Proyectos de Transmisión y Trasformación. ${ }^{3}$ Tales instancias llevan a cabo la planeación, el desarrollo y la operación de los proyectos del sistema eléctrico como parte del permanente proceso de crecimiento de la CFE. ${ }^{4}$ Más adelante se detallará su labor. Pero antes es preciso anotar que el proceso expansivo de la CFE en México tiene décadas: los primeros trabajos de gran envergadura fueron la construcción de presas con fines de generación de energía eléctrica, y el primer antecedente de un trabajo antropológico enmarcado en estos proyectos es el de Alfonso Villa Rojas, quien fue integrado a la Comisión del Papaloapan, cuando se decidió construir la presa Miguel Alemán en la zona mazateca (Villa Rojas, 1948).

De entonces, y hasta bien entrado el siglo XXI, los proyectos de la CFE no estuvieron exentos de conflicto. Los movimientos de resistencia a la construcción de algunas de sus obras fueron estudiados por gente como McMahon (1973), González (1979), García y Medina (1986), Barabás y Bartolomé (1992), Basaldúa (1994), entre otros. Por su parte, la CFE desde la década de 1960 empezó a elaborar en forma estructurada el Estudio de Desarrollo del Mercado Eléctrico, entendido como el análisis de la demanda de potencia y energía y la

\footnotetext{
${ }^{4}$ Se encuentran organizadas de la siguiente manera: Noroeste (Hermosillo, Sonora), Noreste (Monterrey, Nuevo León), Occidente (Guadalajara, Jalisco), Centro (Irapuato, Guanajuato.), Sureste (Veracruz, Ver.) y Peninsular (Mérida, Yucatán), distribuidas de manera estratégica en diferentes zonas de la República Mexicana para una mejor comercialización, con la finalidad de lograr cubrir el territorio nacional en las cuales aproximadamente 1,647 trabajadores están laborando en distintas áreas para dar cumplimiento a la misión de la organización.
} 
elaboración de proyecciones de las mismas. "Al mismo tiempo nació el Programa de Obras e Inversiones del Sector Eléctrico (POISE)..." (CFE, 2014: 2). La estructuración de su misión expansiva podía generar las condiciones para que sus proyectos fueran mejor planificados y consideraran variables como el impacto ecológico y social, aunque la ley no se los exigía.

No fue sino hasta el año 2015, cuando en el marco de la llamada "Reforma Energética", se estableció como requisito legal para cualquier proyecto en materia de generación, transmisión y distribución de electricidad la manifestación de impactos ambientales y la elaboración de EIS. ¿Y cómo tenían que hacerse esas EIS? La responsabilidad de establecerlo estuvo a cargo de la Comisión Federal de Mejora Reguladora-COFEMER- ${ }^{6}$. El 6 de marzo del año 2015, la COFEMER puso en práctica las disposiciones administrativas de carácter general sobre la EIS en el sector energético, como una propuesta de anteproyecto para ser analizada y considerada por la Secretaria de Energía SENER-. La intención era generar un instrumento que funcionara como guía para las instituciones o los promoventes que presentaran un interés de elaboración y desarrollo de proyectos para el impulso del sector energético.

Dado el poco tiempo que ha transcurrió desde esta reforma constitucional a la fecha, no hay hasta el momento proyectos de mediana o gran envergadura emprendidos por otras empresas que no sea la CFE. Esta última ha sido el único promovente interesado en obtener el permiso o la autorización para llevar a cabo proyectos en el rubro eléctrico. Es, entonces, la única que ha tenido que elaborar y presentar EIS ante la SENER, en las cuales se le pide un análisis del área de influencia (el espacio físico que

\footnotetext{
${ }^{5}$ Término empleado para englobar las reformas a la Constitución Política que abrieron la puerta a inversiones privadas en la explotación de petróleo, gas, electricidad y otras formas de energía.

${ }^{6}$ Es un órgano administrativo de alta especialidad en materia de regulación de los procedimientos administrativos de dependencias gubernamentales y
}

probablemente será impactado), incluyendo el área núcleo, área de influencia directa e indirecta.

Los artículos 117 y 120 de Ley de la Industria Eléctrica vigente en México, establecen que todos los proyectos de infraestructura de los sectores público y privado en la industria eléctrica atenderán los principios de sostenibilidad y respeto a los derechos humanos de las comunidades, así como de los pueblos pertenecientes a los lugares donde se pretendan implementar. Igualmente, se les obliga a requerir y conseguir una autorización de parte de la SENER, debiendo presentar una EIS que cumpla con una serie de requisitos, como una identificación, caracterización, predicción y valoración de los posibles impactos sociales que podrían derivarse de sus actividades, así como las medidas de mitigación correspondientes. La EIS deberá ser presentada -dice la ley- en un documento oficial de acuerdo a la guía y el formato que ha establecido la Secretaria para su elaboración, esperando a que emita una resolución en un plazo de 90 días naturales, así como la obtención de los permisos necesarios, tomando en cuenta lo establecido en el artículo 120 de la ley de la industria Eléctrica.

Dado que desde mediados del siglo pasado la CFE priorizó la creación de plantas generadoras de energía (edificando sobre todo centrales hidroeléctricas, termoeléctricas y nucleoeléctricas), actualmente sus proyectos son de nuevas líneas de transmisión y subestaciones que transforman y transmiten la energía eléctrica a lo largo del país. Para este tipo de proyectos, de pequeña o mediana envergadura, la empresa tiene que llevar a cabo EIS por mandato de ley. El último proyecto mayúsculo, encaminado a construir una central hidroeléctrica, fue el de la Presa la Parota, sin embargo, derivó en un conflicto social del que

organismos descentralizados del gobierno federal. La COFEMER cuenta con autonomía y está facultada para emitir un juicio sobre los programas de mejora en las dependencias y organismos descentralizados de la administración pública federal brindándoles asesoría para mejorar sus procedimientos. 
solo se mencionarán algunos elementos para ejemplificar el proceder de la CFE, antes de la obligación legal de elaborar EIS para poner en marcha cualquier proyecto.

La Presa la Parota fue conocido como un megaproyecto ubicado en el sureño estado de Guerrero e involucraba a cinco municipios de esa entidad: Acapulco, Juan R. Escudero, San Marcos, Chilpanlcingo y Tacoanapa. Construir la presa significaba el desalojo de aproximadamente 25000 habitantes y una afectación estimada en 75000 campesinos. Esta Presa se planificó como parte del Plan Puebla Panamá -PPP-, propuesto por el gobierno mexicano en el año 2001 y en el que se involucraron varios países de Centroamérica y el Caribe. Este Plan se proponía facilitar la gestión y ejecución de proyectos orientados a la extracción de recursos naturales de la parte sur de México y de otras naciones de la región. "La Parota sería una de las más de 300 presas programadas de México a Panamá. El objetivo es que generara y acumulara energía eléctrica en cantidades muy grandes para su exportación a través del sistema de Interconexión Eléctrica" (Chávez, 2009: 2).

En este proyecto lo que la CFE buscó inicialmente fue licitar la obra, obtener recursos de la banca internacional e incorporar el proyecto en su programa de obra pública encaminada al desarrollo de la región. El conflicto con los pobladores se ocasionó por la tenencia de las tierras de comuneros y ejidatarios. La obra se comenzó a ejecutar en el año 2003, pero se hizo sin consultar pertinentemente a los pobladores de la región, y mucho menos se les informó de las implicaciones de todo el proyecto. Como respuesta, la sociedad se organizó, movilizó y logró no sólo fortalecer los lazos de fraternidad sino conseguir interlocutores que se identificaron con su lucha, erigiéndose en un movimiento de resistencia emblemático.

Los principales reclamos de la población afectada por el proyecto eran en el sentido de que no solamente se estaban violando sus derechos, sino que había daños ambientales. En este proyecto evidentemente no hubo una
EIS de manera formal, rigurosa, científica. Y aunque hubo una Manifestación de Impacto Ambiental -MIA-, elaborada por colaboradores de la CFE, siempre fue puesta en duda la información por parte de la gente movilizada en los actos de protesta. "El 17 de marzo del 2006, el caso La Parota fue tratado en Primera Audiencia Pública del Tribunal Latinoamericano del Agua (TLA)" (Chávez, 2009). En el veredicto se afirmó que la MIA en ningún momento estaba tomando en consideración los daños que se generarían en torno a la salud pública, ni siquiera a la calidad de vida de la población. El comité de Derechos Económicos, Sociales y Culturales de la Organización de las Naciones Unidas conoció el caso, por medio de la oficina de Alto Comisionado de Naciones Unidas para los Derechos Humanos.

Con antecedentes de este tipo, en los que se planifica una obra, se inician los trabajos y cuando estalla un conflicto social lo que prima es la negociación, la maniobra política o la represión, y no tanto el conocimiento generado mediante un procedimiento riguroso de investigación, como puede implicar una EIS, la CFE batalla contra un prejuicio de parte de la población cuando tiene la intención de poner en marcha nuevos proyectos. Lo anterior no deja de encerrar una paradoja, pues la demanda del servicio de energía eléctrica no se detiene; las poblaciones crecen y con ellas la necesidad de contar con electricidad, pues la vida cotidiana no puede entenderse ya sin ese insumo, pero cuando se trata de aceptar que una subestación se instale en $X$ lugar o que se tiendan líneas de transmisión por Y zona, la resistencia no se hace esperar.

"La CFE se dio a la tarea de construir plantas generadoras en distintos puntos del país contaba ya con 153 plantas para la producción de electricidad y con más de $600,000 \mathrm{~km}$ de líneas de transmisión y distribución" (Secretaria de energía, 2006: 103). Para lograrlo se formularon programas de investigación para responder a la demanda que existía en torno al servicio de energía, lo cual impulsó a la empresa a brindar capacitación de especialistas en todas las fases del mismo, 
extendiendo su cobertura incluyendo, en zonas rurales que habían sido consideradas marginadas, una puerta que permitió la entrada a las ciencias sociales, especialmente a la Antropología. De modo que, el reto al que se ha enfrentado actualmente es conseguir que estos proyectos sean socialmente aceptables sin causar daños permanentes.

\section{Los proyectos de subestaciones eléctricas en Silao, Guanajuato, México}

Este apartado tiene el propósito de describir cómo es que la CFE ha organizado las actividades para atender a la obligación legal de realizar EIS en cada uno de sus proyectos. Se nutre de datos recogidos como parte de una experiencia directa en tal actividad. En primer lugar, hay que decir lo siguiente: dentro del organigrama general de la empresa existe una oficina que se llama Residencia de Actividades Previas, que es la responsable de las EIS. Dentro de esta oficina hay varias áreas que se detallan a continuación:

a) Área de selección del sitio. Ya sea que el proyecto tenga que ver con Líneas de Transmisión Aéreas y Subterráneas o con Subestaciones, su trabajo se centra en considerar las características generales del proyecto, así como la topología actual y futura de interconexión con la red de transmisión e incluso con otras líneas con las que se asociarán. Por cada obra se evalúan tres alternativas para la selección del sitio, con la finalidad de no afectar un área social ambiental y geológica. Considerando que en los casos de sustitución de SE y LT existentes, en zonas urbanas y las requeridas por otras dependencias y entidades oficiales o empresas privadas en las que se establezca un sitio predeterminado $\mathrm{u}$ obligado, se considerará como una alternativa única.

b) Gestión ambiental. Tiene la facultad de atender los requerimientos por parte de la
Secretaria del Medio Ambiente y Recursos Naturales -SEMARNAT-, de acuerdo a lo establecido en el Reglamento de la ley General del Equilibrio Ecológico y la Protección al Ambiente, en materia de evaluación del impacto ambiental en las obras o actividades que requieren autorización en materia de impacto ambiental y de las excepciones ya sean ampliaciones, modificaciones, sustituciones de infraestructura, rehabilitación, mantenimiento de instalaciones que se encuentren en operación.

c) Gestión arqueológica. A través de personal especializado lleva a cabo una prospección de aspectos arqueológicos en el área del proyecto, con el objetivo de tomar decisiones con respecto a la localización definitiva de los sitios o trayectorias para la implementación del mismo, para que esto sea posible y llevado a cabo se cuenta con una supervisión durante su construcción.

d) Gestión indemnizadora. Su función es identificar los afectados de acuerdo al régimen de tenencia de la tierra al que pertenezcan cuyos predios se encuentran ubicados dentro del derecho de vía de la línea de transmisión, para posteriormente efectuar su localización e iniciar las gestiones para obtener anuencia de paso.

e) Gestión social. Son los encargados de obtener la autorización del o los afectados para implementar el proyecto mediante un documento con el cual se otorga el consentimiento para realizar, en la superficie del terreno que se afecta, los trabajos previos y aquellos que se requieran para la construcción de la línea de transmisión o subestación eléctrica, notificando a los afectados por dónde cruzará la LT y donde se ubicará la SE junto con el monto indemnizatorio.

Gráficamente esta organización se ve como lo muestra la Figura 1. 
Figura 1: Organigrama de la Residencia de Actividades Previas.

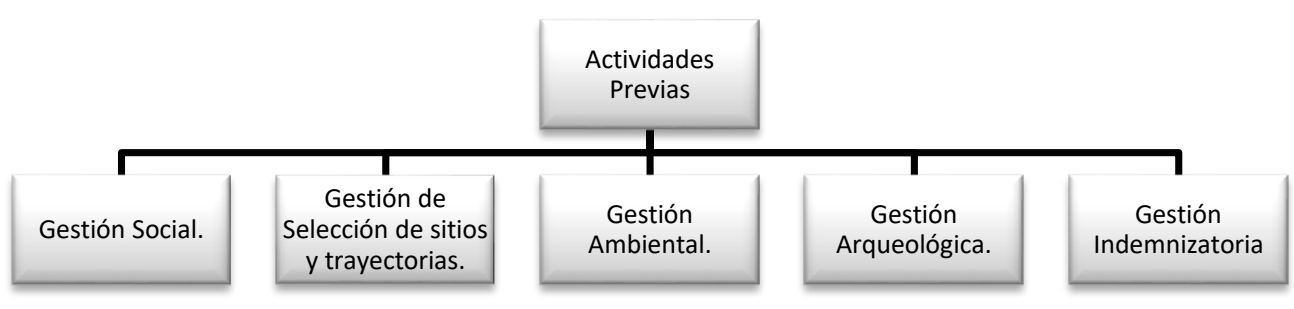

Fuente: Elaboración propia 2018.

Como se dijo desde el principio del artículo, la autora del mismo tuvo oportunidad de participar de manera directa con la CFE para la elaboración de EIS en unas de las primeras subestaciones que se proyectaron tras la nueva obligación legal de ponderar el impacto social.
En el año 2015 en el municipio de Silao, Guanajuato, se llevó a cabo el proyecto PID: 2002 "SLT 2002 Subestaciones y líneas de transmisión Norte Occidental" (Ver Mapa 1 para ubicar la región de trabajo).

\section{Mapa 1}

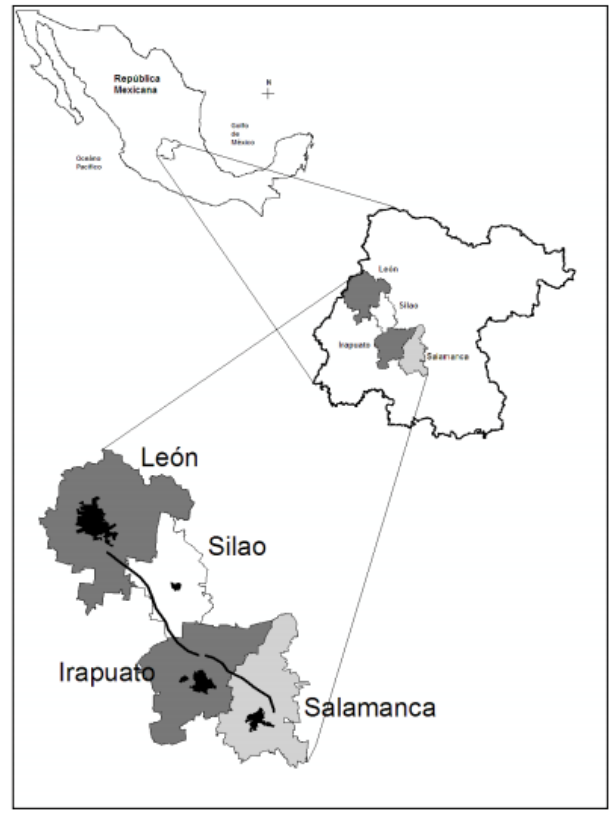

Este proyecto tenía dos propósitos: el primero, proporcionar el servicio de energía eléctrica para uso doméstico en 15 localidades ubicadas en el mencionado municipio y 3 pertenecientes al municipio de León; el segundo, era estar en condiciones de prestar servicios con fines industriales (lo cual requiere un mayor voltaje), dado el crecimiento económico de la zona. La proyección era que se cumplirían los objetivos mediante el enlace tres líneas y subestaciones a cargo de la Residencia
Regional de Construcción de Proyectos de Transmisión y Transformación Centro.

a. Línea de transmisión Silao potencia entq. Romita - Silao I.

b. Línea de transmisión Silao potencia Puerto Interior.

c. Línea de transmisión los Sauces entq. Silao potencia - Puerto Interior. El detalle se aprecia en las tablas 1 y 2. 
Tabla 1

Localidades consideradas en el área de estudio del municipio de Silao.

\begin{tabular}{llcc}
\hline \multicolumn{4}{c}{ Municipio Silao } \\
\hline No. & \multicolumn{1}{c}{ Localidades } & Latitud Norte & Latitud Oeste \\
1 & Lucero de Ramales & $20^{\circ} 55^{\prime} 27^{\prime \prime}$ & $101^{\circ} 27^{\prime} 25^{\prime \prime}$ \\
2 & Condado de la Pila & $20^{\circ} 55^{\prime} 27^{\prime \prime}$ & $101^{\circ} 26^{\prime} 40^{\prime \prime}$ \\
3 & Los Ángeles & $20^{\circ} 52^{\prime} 42^{\prime \prime}$ & $101^{\circ} 23^{\prime} 38^{\prime \prime}$ \\
4 & Bajío de Bonillas & $20^{\circ} 56^{\prime} 58^{\prime \prime}$ & $101^{\circ} 29^{\prime} 43^{\prime \prime}$ \\
5 & Coecillo & $20^{\circ} 58^{\prime} 35^{\prime \prime}$ & $101^{\circ} 26^{\prime} 58^{\prime \prime}$ \\
6 & Col. Nuevo México & $20^{\circ} 59^{\prime} 06^{\prime \prime}$ & $101^{\circ} 28^{\prime} 59^{\prime \prime}$ \\
7 & San Antonio Texas & $20^{\circ} 59^{\prime} 12^{\prime \prime}$ & $101^{\circ} 30^{\prime} 12^{\prime \prime}$ \\
8 & Loza de Barrera & $20^{\circ} 59^{\prime} 05^{\prime \prime}$ & $101^{\circ} 31^{\prime} 47^{\prime \prime}$ \\
9 & Mezquite de Sotelo & $21^{\circ} 00^{\prime} 32^{\prime \prime}$ & $101^{\circ} 30^{\prime} 09^{\prime \prime}$ \\
10 & Nápoles & $21^{\circ} 01^{\prime} 36^{\prime \prime}$ & $101^{\circ} 28^{\prime} 09^{\prime \prime}$ \\
11 & Refugio de los Sauces & $21^{\circ} 00^{\prime} 54^{\prime \prime}$ & $101^{\circ} 31^{\prime} 52^{\prime \prime}$ \\
12 & El Refugio de la Pila & $20^{\circ} 55^{\prime} 45^{\prime \prime}$ & $101^{\circ} 26^{\prime} 33^{\prime \prime}$ \\
13 & Franco & $20^{\circ} 56^{\prime} 33^{\prime \prime}$ & $101^{\circ} 27^{\prime} 03^{\prime \prime}$ \\
14 & La Aldeíta (la Cotorra) & $20^{\circ} 54^{\prime} 35^{\prime \prime}$ & $101^{\circ} 28^{\prime} 19^{\prime \prime}$ \\
15 & Fracc. San Juan de los Durán & $20^{\circ} 54^{\prime} 49^{\prime \prime}$ & $101^{\circ} 27^{\prime} 05^{\prime \prime}$ \\
\hline
\end{tabular}

Fuente: Elaboración propia D.S. 2015.

Tabla 2

Localidades consideradas en el área de estudio del municipio de León.

\begin{tabular}{|c|c|c|c|}
\hline \multicolumn{4}{|c|}{ Municipio León } \\
\hline No. & Localidades & Latitud Norte & Latitud Oeste \\
\hline 1 & Albarradones & $21^{\circ} 02^{\prime} 26^{\prime \prime}$ & 101³0'14" \\
\hline 2 & San José de los Romeros & $21^{\circ} 02^{\prime} 16^{\prime \prime}$ & $101^{\circ} 29^{\prime} 30^{\prime \prime}$ \\
\hline 3 & Lomas de Comanjilla & $21^{\circ} 01^{\prime} 59^{\prime \prime}$ & $101^{\circ} 31^{\prime} 47^{\prime \prime}$ \\
\hline
\end{tabular}

Fuente: Elaboración propia D.S. 2015.

Durante el trabajo de campo realizado en todas estas comunidades se pudo constatar que en cada una de ellas el servicio eléctrico es demandado por la población, debido a que la electricidad se ha vuelto necesaria en la vida de las personas, agilizando las actividades que llevan a cabo estas en sus hogares, escuelas, lugar de trabajo, en la calle, etc. La totalidad de las localidades cuentan con servicio eléctrico y lo demandan de manera creciente. Pese a esto, la desconfianza, el recelo y la resistencia se manifiestan cuando la CFE se hace presente en los sitios buscando promover algún proyecto para mejorar sus condiciones de prestación del servicio.
No fue difícil darse cuenta que gran parte de la población de estas localidades no tiene un buen concepto de la empresa. Ello se debe a información que se ha propagado por distintos medios de comunicación (televisión, redes sociales, periódico, radio, entre otros) vinculada a casos como el ya referido proyecto de La Parota. La situación se agrava cuando el personal de la CFE (o algún subcontratista) se acerca a algunos pobladores para plantearles la idea de vender su tierra, o por lo menos rentarla, para establecer una subestación o que pasen por ahí líneas de transmisión.

Cualquier persona que se ve precisada a dejar su tierra y reubicarse, verá transformada su vida, para bien o para mal. Potencialmente esta 
situación puede derivar en conflictos sociales que pongan en riesgo al proyecto mismo. ¿Cómo solucionar estos conflictos? Si la sociedad necesita el servicio, pero no quiere más intromisión y daños en su entorno, ¿las EIS tendrían que limitarse a proyectar las afectaciones?, ¿deberían buscar alternativas para realizar los proyectos y mitigar las afectaciones? Este era un reto que debían asumir los antropólogos en campo. ¿Y cómo hacerlo?, ¿Qué tanta distancia epistemológica, deontológica y política existe respecto de lo propuesto por Villa Rojas, quien en 1948 asumió la dirección de la Sección de Antropología de la Comisión del Papaloapan, encargada de gestionar el proyecto de la presa Miguel Alemán? Rojas (1948) definió así su tarea:

Conocer en términos sociológicos la realidad social existente en lo que forma la Cuenca del Papaloapan, a fin de tener así una guía que permita orientar la acción planificada de modo tal que no provoque estados de tensión, conflictos ni relaciones adversas en el medio social que se trata de transformar. Para ello nos proponemos investigar de modo sistemático, los usos, costumbres, hábitos, valores y todo cuanto dé forma y sentido a los modos de vida de esta región (p. 14).

Investigar y llegar a conocer a la gente y sus modos de vida ¿para qué?, ¿Se trata de ocupar esta información para saber cómo manipular sus voluntades?, ¿El asunto es conocer para proyectar qué tanto se vería afectada su vida y costumbres con un cambio como su reubicación territorial?, ¿Conocerlo es solo el paso inicial necesario para dialogar?, ¿El diálogo sería el camino para buscar que el proyecto se desarrolle y que la vida en la región encuentre cause?

Dada la casi nula experiencia en materia de EIS dentro de la CFE, para atender esta nueva necesidad ha incorporado a su Residencia de Actividades Previas a algunos especialistas, a quienes se les encarga implementar sus conocimientos, metodologías y estrategias para llevar a cabo los estudios. Los antropólogos sociales son algunos de estos especialistas recientemente incorporados, que se suman a biólogos, geógrafos, sociólogos, ingenieros civiles, arquitectos, abogados, topógrafos, entre otros. Una de las funciones del antropólogo es apoyar en el proceso de selección de sitios para realizar la obra, y en el caso del proyecto ubicado en Silao, la parte técnica ofreció tres opciones dentro del área de selección (las cuales suelen ubicarse vía remota, con fotografías satelitales e información cartográfica), así el antropólogo tenía la responsabilidad de ir a campo, estar a nivel de tierra y ponderar cuál era la mejor opción.

Desde luego también se suele realizar trabajo de gabinete, recopilando información on-line, información publicada en prensa e incluso en redes sociales, para contar con antecedentes relevantes de cada comunidad que se encuentre en el área de influencia del proyecto. Después era preciso efectuar un enlace comunitario; esto quiere decir acercarse a las personas, tener una interacción con ellos y buscar que ellos se conviertan en partícipes del proyecto, que se sientan actores interesados por el mismo.

Por así requerirlo la guía de las EIS, el antropólogo debe recopilar información que incluirá en el reporte del Diagnostico Social Previo a la EIS. Se le recomienda realizar un análisis demográfico para conocer los elementos e indicadores que conformarán la línea base: tamaño, estructura y distribución de la población, situación política, social, seguridad, salud, vivienda, economía, religión, educación, tradiciones, cosmovisión, entre otras; todo esto con la intención de asegurarse $\mathrm{y}$ asegurarle a su equipo de trabajo que desde su punto de vista se está tomando la decisión correcta al seleccionar, por ejemplo, la ruta que debe tener una línea de transmisión. Él no es el único que tiene que llevar a cabo una serie de tareas para llegar a este resultado, cada área encargada de analizar las rutas también presenta un informe, justifican la decisión que han tomado y que puede ser considerando la flora, la fauna, los cuerpos de agua, etc. Al 
reunir los elementos necesarios y al estar en total acuerdo se define qué trayecto seguir.

En el caso de la labor que como antropólogo se realizó, la visita al campo permitió tener el acercamiento con las personas y así darse cuenta que cierta información, que ya poseía la CFE y que era considerada "de primera mano" (como el tamaño de la comunidad, por ejemplo), no se encontraba actualizada, esto implicaba proyectar de manera errónea el porcentaje de población que podía ser impactada. Así, de no haberse documentado de mejor manera el territorio y las actividades económicas del lugar, las decisiones tomadas seguramente hubieran sido inadecuadas. Así mismo, en algunas localidades de las que involucró el proyecto la tierra es el medio de subsistencia o, por lo menos, un referente central en la cosmovisión de las personas, de modo que proponerles la compra, puede ser automáticamente rechazado, pues a esta se le ha atribuido un significado importante que forma parte de la cultura intangible de sus pobladores.

Aunque en el caso del proyecto de Silao no había localidades con presencia mayoritariamente indígena, sí se comentaba entre los equipos de trabajo que se han presentado casos en los cuales, en el área de influencia de los proyectos a desarrollar, se ubica un porcentaje alto de presencia de poblaciones indígenas ( $40 \%$ o más); al detectar este elemento, el Antropólogo tiene que realizar no solo la EIS, sino también la Consulta a los Pueblos Indígenas -CPI- que prevé la ley. Esta consulta se volvió obligatoria también a partir de la "Reforma Energética". Entonces, al detectarse que mucha de la información documental sobre las comunidades ya no se correspondía con la realidad, fue necesario realizar un estudio etnográfico que arrojara información más acertada. Así, El acercamiento con las personas permitió, además, establecer estrategias de coordinación con líderes y agrupaciones sociales locales, algunas de índole religioso, para hacer extensivas a la población las características del proyecto, y donde siempre se buscaron alternativas para que se comprendiera plenamente el proyecto $\mathrm{y}$ no causar descontento.

Cabe recordar que toda esta actuación fue conducida bajo el Plan de Gestión Social que emitió la COFEMER y que se define como:

la estrategia e implementación del conjunto de medidas de ampliación de impactos positivos, así como la prevención y mitigación de los impactos negativos. Se define y se programa con la participación de los actores interesados y se realiza por el promovente (COFEMER, 2015: p 5).

\section{Reflexiones finales}

Este trabajo buscó describir la manera en la que se empiezan a implementar las EIS en México por parte de la CFE a partir de una obligación legal. El trabajo realizado en los proyectos de la CFE en el estado de Guanajuato, de los que ya se han dado detalles, son de los primeros en los que se aplica la nueva normatividad que obliga a efectuar EIS. Los documentos guía que han sido elaborados por la COFEMER han hecho de esta labor una especie de trámite burocrático a cubrir, por tanto, esta visión no abona en nada a la construcción de un cuerpo sólido de conocimiento y metodología para hacer de las EIS un medio de gestión de los proyectos $\mathrm{y}$, al mismo tiempo, plantean una disyuntiva a deontológica a los antropólogos que se han visto involucrados; tener empleo es suficiente razón para avalar este "trámite burocrático", o debe apostarse por empujar una reflexión epistemológica y procedimental para hacer de las EIS un instrumento de utilidad para todos los actores que se verán involucrados en el proyecto.

Es conveniente para los antropólogos sociales, que son incorporados en calidad de especialistas, ir empujando la idea de que las EIS pueden llegar a ser de mayor utilidad, sí se les da el sentido de ser el medio para que los proyectos incorporen a la población, y esto se debe lograr desde el modelo dialogal de las EIS. Este modelo, que han cultivado desde la 
hoy conocida como SIA, considera que la labor del que hace EIS no es un servicio para el promovente, ni tampoco se trata de una apuesta en defensa del afectado, y no solo debe rendir cuentas a las autoridades. El antropólogo social, puesto en la condición de hacer EIS, debe desarrollar capacidad de dialogar con los tres: la CFE, la población y la autoridad. De modo que, debe traducirle a cada uno de ellos lo que el otro propone, piensa o necesita, debe mediar y proponer soluciones en las que todos puedan transigir, aceptar, ofrecer y aceptar del otro. Esto es necesario porque la dinámica de empresas como la CFE es continuar expandiéndose y no dejar de impulsar proyectos, entonces, es necesario encontrarles causes de salida a los mismos.

Para conseguir esta posición el antropólogo no sólo debe manejar con experticia la legislación aplicable y los procedimientos administrativos necesarios, sino también debe dominar pertinentemente temas como sustentabilidad, derechos humanos, género, comunidad, justicia, o resiliencia, entre otros, al mismo tiempo, debe conservar su sensibilidad y empatía para entender a los pobladores y su modo de ver el mundo y vivir la vida. La tarea de evaluación de impacto social no se agota en el llenado de los formularios y el otorgamiento del aval que la autoridad requiere, la suya, es una labor científica anclada en una epistemología dialogal, donde los saberes se comportan como sistemas ecológicos; conviven (no siempre de modo armonioso e incluyente) el saber técnico del geógrafo, del ingeniero y del administrador con los saberes ancestrales de los comuneros y ejidatarios, igualmente en su derredor están los saberes jurídicos, económicos y demás, en tanto el antropólogo puede verlos a todos de manera holística. No solo se evalúa qué perjuicios traerá un proyecto, también se evalúa cómo es la vida en la comunidad impactada y de qué manera puede insertarse en ella un proyecto planificado.

\section{Referencia}

Barabas, A. y Bartolomé, M. (1986). Desarrollo hidráulico y etnocidio: los pueblos mazateco y chnanteco de Oaxaca". En García y Medina (Ed.), La quiebra política de la antropología social en México Vol. II La polarización (19711976) (pp. 353-367). México: UNAM.

Barabas, A. y Bartolomé, M. (1992). Antropología y relocalizaciones. Alteridades, 2(4), pp. 5-15.

Basaldúa, M. (1994). La respuesta política de la población de Villahermosa al proyecto hidroeléctrico de Zimapán. (Tesis de Maestría en Antropología Política), Universidad Autónoma de Querétaro, México.

Becker, H. (1997). Evaluación del impacto social: método y experiencia en Europa, América del Norte y el mundo en desarrollo, Londres: UCL.

Burdge, R. y Vanclay, F. (1995). Social impact assessment. En Vanclay y Bronstein (Ed.), Environmental and social impact assessment (pp. 3166). Chichester: Wiley.

Chávez-Galindo, R. (2009). El conflicto Presa la Parota, Guerrero, Méx.: Estado del Desarrollo Económico y Social de los Pueblos Indígenas de Guerrero Programa Universitario México Nación Multicultural. México: UNAM. Recuperado de http://www.nacionmulticultural.unam.m x/edespig/diagnostico_y_perspectivas/R ECUADROS/CAPITULO $\% 2010 / 3 \% 20$ el\%20conflicto\%20presa\%201a\%20paro ta.pdf

Comisión Federal de Electricidad. (2014). Programa de Obras e Inversiones del Sector Eléctrico, POISE 2014-2028.

Recuperado de https://www.amdee.org/Publicaciones/P OISE-2014-2028.pdf

Comisión Federal de Electricidad. (2015). Evaluación de Impacto social. PID 1820, Irapuato, Gto: CFE. 
Comisión Federal de Electricidad. (2018). Página oficial de la CFE. Recuperado de https://www.cfe.mx/Pages/Index.aspx

Comisión Federal de Mejora Regulatoria. (2015). Disposiciones administrativas de carácter general sobre la Evaluación de Impacto Social en el Sector Energético. Recuperado de http://www.cofemersimir.gob.mx/exp edientes/14565

CONEVAL (2014). Metodología para la aprobación de indicadores de los programas sociales. México: Consejo Nacional de Evaluación de la Política de Desarrollo Social.

Esteves, Ana Maria, Daniel Franks y Frank Vanclay (2012) Evaluación de impacto social: el estado del arte. Evaluación de impacto y evaluación de proyectos, 30 (1), p. 34-42.

Foster, G. (1964). Las culturas tradicionales y los cambios técnicos. México: Fondo de Cultura Económica.

García, E. \& Cardozo, M. (2017). Evaluación de impacto: más allá de la experimentación. Política y cultura, (47), 65-91. Recuperado en 19 de febrero de 2019, de http://www.scielo.org.mx/scielo.php? script=sci_arttext\&pid=S018877422017000100065\&lng=es\&tlng=es

García, C. (2007). Evaluación económica de un sistema de tratamiento de aguas residuales para una central termoeléctrica propiedad de la C.F.E., (Tesis de Maestría en Ciencias con Especialidad en Ingeniería Industrial). Instituto Politécnico Nacional, México D.F.

Gómez-Serra, M. (2004). Evaluación de los servicios sociales. Barcelona, España: Gedisa.
González, N. (1979). La Sociología de una Presa, Traducción de R. Melville. Cuadernos de Posgrado en Antropología Social, (1), pp. 34-60

Instituto Nacional de Estadística y Geografía. (2015). Principales resultados de la Encuesta Intercensal 2015, Estados Unidos Mexicanos. Recuperado de http://internet.contenidos.inegi.org.mx/c ontenidos/Productos/prod_serv/contenid os/espanol/bvinegi/productos/nueva_est ruc/702825078966.pdf

Kisnerman, N. (1984). Teoría y Práctica del Trabajo Social-El Método de Intervención-Transformación. Argentina: Humanitas.

Malinowski, B. (1929) Practical Anthropology, Africa, 2 (1), 22-38.

McMahon, D. (1973). Antropología de una Presa: Los Mazatecos y el Proyecto de Papaloapan, N. México: Consejo Nacional para la Cultura y las Artes.

Pichardo, A. (2013). Evaluación del impacto social. Argentina: Lumen.

Secretaria de Energía. (2006). Energía: compromiso para el desarrollo social y económico de México. México, D.F.: Fondo de Cultura Económica.

Serrano, J. M. (2011). Análisis y gestión de riesgo en el mantenimiento de un sistema eléctrico, caso de: Una Subestación de alta tensión. (Tesis de Maestría en Ciencias en Ingeniería de Sistemas). Instituto Politécnico Nacional, México, D.F.

Stufflebeam, D.L. Y Shinkfield, A.J. (1987). Evaluación sistemática. Guía teórica y práctica. Madrid: Paidós-MEC. 
Tenbrink, T.D. (1988). Evaluación: guía práctica para profesores. Madrid: Marea.

Vanclay, F. (1999). Evaluación de impacto social. Manual de evaluación de impacto ambiental, 1, 301 - 326.

Vanclay, F. (2003). Principios internacionales para la evaluación del impacto social. Evaluación de Impacto y Evaluación del Proyecto, 21 (1), $5-11$.

Villa Rojas, A. (1948). Antropología de una Presa: Los Mazatecos y el Proyecto de Papaloapan, N. México.

Villa Rojas, A. (1948a). En: McMahon, D (1973). Antropología de una Presa: Los Mazatecos y el Proyecto de Papaloapan, INI: México. Consejo Nacional para la Cultura y las Artes

Xolo-Pucheta, R. (2011). Propuesta Metodológica para la planificación de los proyectos de construcción a través del módulo de PS del SAP R/3, caso: Residencia Regional Sureste de la CFE, (Tesis de Maestría en Administración de la Construcción). Instituto Tecnológico de la Construcción, Veracruz, México. 